\title{
Synthesis of Silver Nanoparticles by Leaf Surface Fungi Isolated from Piper betle L. and its Antimicrobial Potential Study ${ }^{\dagger}$
}

\author{
Bijaya Kumar Nayak ${ }^{1,}{ }^{*}$, Nithiya G. ${ }^{1}$, Anima Nanda ${ }^{2}$ \\ 1 Department of Botany, Kanchi Mamunivar Govt. Institute for Postgraduate Studies and Research (Autonomous), \\ Puducherry-605008 \\ 2 Department of Biomedical Engineering, Sathyabama Institute of Science and Technology, Deemed to be University, \\ Chennai- 600119 \\ * Correspondence: bijuknayak@gmail.com; \\ $\uparrow \quad$ Presented at International e-Conference on Bioengineering for Health and Environment (ICBHE 2020)
}

Received: 5.07.2020; Revised: 10.07.2020; Accepted: 12.07.2020; Published: 15.07.2020

\begin{abstract}
Studies on the biodiversity of phylloplane and endophytic fungi on the leaf surfaces of the medicinal plant; Piper betle L. was made in our Microbiology laboratory, K.M. Govt. Institute for Postgraduate Studies and Research (Autonomous), Puducherry. The Agar plate method was used to isolate both the leaf surface and sub-surface fungi. During the study period, altogether twelve fungal species of seven genera of phylloplane and five species of endophytes were isolated from Piper betle L. by agar plate method. Penicillium sp., a dominant fungus, was chosen for the synthesis of silver nanoparticles. Silver nanoparticles (AgNPs) were synthesized from Ag+ ions by treating with different extracts of the fungus with $\mathrm{AgNO}_{3}$. The appearance of yellowish-brown color in the conical flasks suggested the formation of AgNPs in dark and light conditions. The AgNPs were characterized by UVVis spectroscopy, which has proved to be very useful for the analysis of nanoparticles. Candida albicans was found most susceptible towards the AgNPs of the fungus in comparison to other bacterial strains. Among the bacterial strains, Staphylococcus aureus was more suffered than V. parahaemolyticus and $E$. coli. It was found in the present study that our process for the synthesis of nanoparticles was easy, safe, and economical to be readily used in the field of biomedicine.
\end{abstract}

Keywords: Silver nanoparticles; Leaf surface fungi; Antimicrobial potential; Candida albicans; Biomedicine.

(C) 2020 by the authors. This article is an open-access article distributed under the terms and conditions of the Creative Commons Attribution (CC BY) license (https://creativecommons.org/licenses/by/4.0/).

\section{Funding}

This research received no external funding.

\section{Acknowledgments}

This research has no acknowledgment.

\section{Conflicts of Interest}

The authors declare no conflict of interest. 